\title{
Water flow enhancement in amorphous silica nanochannels coated with monolayer graphene
}

Wagemann, Enrique; Becerra, Diego; Walther, Jens H.; Zambrano, Harvey A.

Published in:

M R S Communications

Link to article, DOI:

$10.1557 / \mathrm{mrc} .2020 .53$

Publication date:

2020

Document Version

Peer reviewed version

Link back to DTU Orbit

Citation (APA):

Wagemann, E., Becerra, D., Walther, J. H., \& Zambrano, H. A. (2020). Water flow enhancement in amorphous silica nanochannels coated with monolayer graphene. M R S Communications, 10(3), 428-433.

https://doi.org/10.1557/mrc.2020.53

\section{General rights}

Copyright and moral rights for the publications made accessible in the public portal are retained by the authors and/or other copyright owners and it is a condition of accessing publications that users recognise and abide by the legal requirements associated with these rights.

- Users may download and print one copy of any publication from the public portal for the purpose of private study or research.

- You may not further distribute the material or use it for any profit-making activity or commercial gain

- You may freely distribute the URL identifying the publication in the public portal

If you believe that this document breaches copyright please contact us providing details, and we will remove access to the work immediately and investigate your claim. 


\title{
Water flow enhancement in amorphous silica
}

\section{nanochannels coated with monolayer graphene}

\author{
Enrique Wagemann, ${ }^{\dagger}$ Diego Becerra, ${ }^{\ddagger}$ Jens $\mathrm{H}$. Walther, ${ }^{\boldsymbol{9}}, \S$ and Harvey A. \\ Zambrano*,\| \\ $\dagger$ Departamento de Ingeniería Mecánica, Facultad de Ingeniería, Universidad de \\ Concepción, Concepción, Chile \\ $\ddagger$ Departamento de Ingeniería Química, Facultad de Ingeniería, Universidad de Concepción, \\ Concepción, Chile \\ ITechnical University of Denmark, Copenhagen, Denmark \\ §Chair of Computational Science, ETH Zurich, Zurich, Switzerland \\ ||Department of Mechanical Engineering, Universidad Técnica Federico Santa María, \\ Valparaiso, Chile
}

E-mail: harvey.zambrano@usm.cl 


\begin{abstract}
Inspired by the recently reported translucency of monolayer graphene to wetting, atomistic simulations are employed to evaluate water flow enhancement induced by graphene deposited on the inner surfaces of hydrophilic nanochannels. The flow in the coated channels exhibit a slip length of ca. $3.0 \mathrm{~nm}$. Moreover, by contrasting the flow rates in channels with coated walls against flow rates in the corresponding uncoated channels, an "effective" flow enhancement from 3.2 to 3.7 is computed. The PDF of the water dipole orientation indicates that the flow enhancement is related to a thinner structured water layer at the solid-liquid interface. This study provides quantitative evidence that graphene employed as coating reduces substantially hydraulic losses in hydrophilic nanoconfinement.
\end{abstract}

\title{
Introduction
}

Fast-growing advances in fabrication techniques are enabling further miniaturization of microfluidic systems. This length scale reduction has led to the emergence of a new technological field, i.e., nanofluidics. ${ }^{1}$ For water confined inside nanoscale hydrophilic conduits, recent investigations have reported extremely low flow rates. This phenomenon has been associated to very large viscous shear forces at solid-liquid interfaces. ${ }^{2,3}$ Indeed, giant hydraulic resistances in hydrophilic nanochannels have been attributed to interfacial molecular ordering and size dependent shear viscosity. ${ }^{3}$ Hence, drag reduction is a key technological problem related to the development of efficient nanoscale fluidic devices.

Recent studies have reported ultra fast motion of nanoconfined water in contact with hydrophobic walls, i.e., higher than expected water flow rates in carbon nanotubes (CNTs) and graphene (GE) nanochannels. ${ }^{4-6}$ In fact, experiments and computational studies have provided evidence that these high flow rates strongly depend on the ultra-low friction at the carbon-water interface that translates into very large slip lengths. ${ }^{4,7-9}$ This low friction of water in contact with CNT walls and GE sheets has been related to their atomically smooth 
graphitic surfaces and to their weak interaction with water. ${ }^{8,10}$ Consequently, GE layers and CNTs are promissory candidates to be used as fluid conduits or as ultra thin wall coatings inside hydrophilic nanopores to decrease the amount of energy required to transport fluid across nanodevices and nanostructured membranes. ${ }^{1,11}$

The intrinsic wetting properties of GE surfaces have been a subject of intense study over the last decade. ${ }^{7,8,12,13}$ Indeed, recent investigations examining the degree to which the underneath substrate affects the water contact angle (WCA) on supported GE sheets ${ }^{14-17}$ have brought water wettability of GE to intense debate. ${ }^{13}$ In fact, those studies reported either full transparency to wettability ${ }^{18}$, translucency ${ }^{14,18-21}$ or even total opacity ${ }^{22,23}$ for water in contact with GE supported on different type of substrates. In particular, for monolayer GE supported on a silica substrate, Rafiee et al. ${ }^{18}$ found wetting translucency, reporting a WCA of $49^{\circ}$. For graphitic materials, previous computational studies have already investigated the effect of the translucency to wettability of GE sheets over the slippage in coated substrates. ${ }^{24-26}$ However, those investigations focused on systems wherein the underlying substrates consisted of crystalline structures coated by perfectly planar GE layers. Moreover, in their studies the viscous heat was not removed through the walls of the system due to the positions of the atoms in the solid material were kept fixed during their simulations. It should be noted that maintaining frozen the positions of the wall atoms can result in unphysical behavior of the interfacial water and might artificially alter slippage on the solid surface within the channel. ${ }^{27}$ Additionally, surface roughness is known to affect the slippage at the solid-liquid interface. ${ }^{9,28}$ These factors are considered in the present study and special care is taken upon them. Here, we employ MD simulations to investigate whether the use of a GE monolayer as wall coating inside a hydrophilic silica channel may have a significant impact on water flow. Our results provide quantitative evidence that GE coating in a silica nanochannel results in substantial drag reduction in water transport which can implications for the design and fabrication of efficient integrated nanofluidic devices. 


\section{Computational methods}

The atomistic simulations in this work are carried out using the parallel MD package FASTTUBE, which has been used extensively to study liquids confined inside CNTs, GE layers and silica channels. ${ }^{6,9,29}$ The water molecules are described using the rigid SPC/E water model. ${ }^{30}$ The silica atoms are described using the TTAMm potential developed by Guissani and Guillot ${ }^{31}$ which is a modification of the classical TTAM potential developed by Tsuneyuki et al.. ${ }^{32}$ In this study, we employ the original set of atomic partial charges in the TTAM model which correspond to values of: $q_{S i}=+2.4 e$ and $q_{\mathrm{S}_{\mathrm{Si}_{2}}}=-1.2 e$. The van der Waals interactions between silica atoms and water molecules are modeled using a Buckingham potential with parameters taken from our previous work. ${ }^{33}$ The GE-water interactions are described by a Lennard-Jones potential parametrized by Werder et al. ${ }^{34}$ to reproduce a macroscopic water contact angle (WCA) on graphite of $86^{\circ}$. The GE-silica interactions are described employing a Lennard-Jones potential with parameters from Zhang and $\mathrm{Li}^{35}$ which reproduce the proper substrate-regulated morphology of a supported GE layer. The carbon-carbon valence forces within the GE sheet are modeled using Morse, harmonic angle and torsion potentials. ${ }^{29}$ To validate our atomistic models, MD simulations are performed of a cylindrical nanodroplet of water on a GE coated substrate. The cylindrical shape of the nanodroplet allow us to measure directly the experimental macroscopic WCA of $86^{\circ 18}$ avoiding the line tension effect linked to spherical nanodroplets. For more details about the WCA simulations, employed protocols and atomistic models, readers are referred to the supporting information.

\section{Results and Discussion}

To study the flow enhancement induced by GE coating the walls of a hydrophilic nanochannel, Poiseuille like flow of water is simulated in an amorphous silica nanoslit channel with and without monolayer GE monolayer coating the inner silica surfaces. The nanoslit consists 
of two parallel slabs of amorphous silica, representing the channel walls. A single sheet of GE is deposited on the inner surface of each silica wall. A snapshot of the system is shown in Figure 1 A. The channels have a length (L) of $11.06 \mathrm{~nm}$ and a width (w) of $3.50 \mathrm{~nm}$. Channel with three heights (h) are studied here; 2.4, 3.4 and $4.4 \mathrm{~nm}$. The water density profiles are shown in Figure 1 B, displaying bulk and interfacial densities in good agreement with values reported in previous works. ${ }^{36}$ Furthermore, the computed density profiles display water layering at the solid-liquid interface with thickness of ca. $0.85 \mathrm{~nm}$. As depicted in Figure $1 \mathrm{~B}$, the layering thickness is the same for all the cases studied here, hence it does not depend on the particular height of the channel. Nevertheless, as confinement increases, the ratio between layering thickness and the channel height increases altering the effective viscosity in the system ${ }^{37}$.

Fluid flow is generated by imposing a constant external field that acts parallel to the channel axis and is applied to all water molecules confined inside the channel. We impose constant external field ranging from $2.331 \times 10^{11}$ to $9.324 \times 10^{11} \mathrm{~m} / \mathrm{s}^{2}$. These external fields correspond to pressure gradients ranging from $2.26 \mathrm{bar} / \mathrm{nm}$ to $9.04 \mathrm{bar} / \mathrm{nm}$. As shown in Figure $1 \mathrm{C}$, the volumetric flow rates $(Q)$ are found to depend linearly on the magnitude of the applied external field. The flow enhancement $(\epsilon)$ is computed as the ratio between the measured water flow rates and the corresponding theoretical no-slip Poiseuille flow rates $\left(Q_{H P}\right)$. The volumetric flow rates for Poiseuille like flow with no-slip boundary condition are calculated using,

$$
Q_{H P}=\frac{h^{3} w \Delta P}{12 \mu_{\infty} L}=\frac{h^{3} w \rho g}{12 \mu_{\infty}}
$$

where $\rho$ corresponds to the overall fluid density in the channel, $g=\frac{d u_{y}}{d z}$ is the applied external field and $\mu_{\infty}$ is the viscosity of SPC/E water in bulk $(0.729 \mathrm{mPa} \cdot \mathrm{s})^{38}$. The channel height (h) is computed as the average distance between the carbon atoms in the two GE sheets coating the channel walls, subtracting the van der Waals size of the atoms $(0.34 \mathrm{~nm})$. Increasing $\epsilon$ values of ca. $6.0 \pm 0.1,7.8 \pm 0.2$ and $11.3 \pm 0.5$ are computed for channels with decreasing 
heights of $4.4 \mathrm{~nm}, 3.4 \mathrm{~nm}$ and $2.4 \mathrm{~nm}$, i.e., wherein a higher ratio of surface to confined fluid volume is present. Hence, the computed $\epsilon$ values confirm that GE coatings have a stronger effect on the flow of water inside channels with higher confinement and indicate that the implementation of monolayer GE coatings in amorphous silica nanochannels results in a substantial mitigation of the hydrodynamic resistance and thus in an effective decrease in the energy required for water transport. In line with previous studies, our results show that in nanoconfined Poiseuille flow with finite slip, flow enhancement is directly related to a reduction in the viscous drag. ${ }^{3}$ Moreover, $\epsilon$ values around 10 for water flow inside a GE coated silica channel imply that similar flow rates can be attained imposing a pressure gradient one order of magnitude lower than expected for a hydrophilic pristine channel.

Figure 2, shows the velocity profiles for all the cases studied here. The velocity profiles exhibit a parabolic shape with a significant wall slippage, i.e., non-zero flow velocities are computed at the solid-liquid interface, as found in previous studies of water flow nanoconfined between graphitic walls. ${ }^{4,6,9}$ Moreover, we compute the slip length $\left(l_{s}\right)$ in the channels studied here, by imposing a parabolic fit to the corresponding velocity profiles. These values are listed in Table S4 of the supporting information. A constant slip length of ca. $3.0 \mathrm{~nm}$ is observed for all the cases, which indicates that $l_{s}$ is not dependent on the channel height. The computed $l_{s}$ is considerably lower that the one for free standing graphene $\left(\mathrm{ca} .50 \mathrm{~nm}^{6}\right)$, suggesting an substantial effect of the underlying substrate on the friction imposed by the coated nanochannel. Moreover, the computed $l_{s}$ is lower than the one reported by previous works that have assesed the flow enhancement due to the use of graphene as coatings in nanochannels ${ }^{25,26}$. Such difference stems from the difference in the surface roughness and hydrophilicity of the studied underlying substrates (our substrate is more hydrophilic and has higher surface roughness). We estimated the effective viscosity ${ }^{37}\left(\mu_{e f f}\right)$ for each channel studied here based on the Newton's law of viscosity:

$$
\tau=\mu_{e f f} \frac{d u_{y}}{d z}
$$


where $\frac{d u_{y}}{d z}$ is computed from a parabolic fit to the velocity profile. Note that values obtained of ca. $0.654 \mathrm{mPa} \cdot \mathrm{s}, 0.662 \mathrm{mPa} \cdot \mathrm{s}$ and $0.658 \mathrm{mPa} \cdot \mathrm{s}$, presented in Table $\mathrm{S} 4$ of the supporting information, indicate a constant $\mu_{e f f}$, with values that are lower than the corresponding values of $\mu_{\infty}$. The difference between $\mu_{\text {eff }}$ and $\mu_{\infty}$ suggests that the GE coating induces a decrease in the shear viscosity of the fluid. ${ }^{36}$

It should be noticed that in nanoconfined geometries, water is known to slip on both hydrophilic and hydrophobic surfaces. ${ }^{39}$ Hence, values of flow enhancement computed by a direct comparison between the measured flow rates against the theoretical flow rate calculated with no-slip boundary condition could not be a proper criterion to estimate the actual reduction of the hydrodynamic resistance in a coated nanochannel. Moreover, for nanofluidic applications, it is key to understand in a realistic scenario the drag reduction capabilities of GE coatings. Hence, note that in a nanochannel, drag reduction achieved by coating the inner walls is not exempt of cost in terms of changes in the channel geometry. In fact, the presence of a GE sheet on the channel walls may decrease significantly the cross section of the nanochannel, an effect that can increase significantly the hydrodynamic resistance, thus, reducing the flow-enhancing capabilities of GE wall coatings in a realistic scenario. In order to take these factors into account, we measure flow rates of water confined inside nanoslit silica channels with uncoated walls from the atomic trajectories. Subsequently, we calculate the "effective" flow enhancement as the ratio between the flow rates measured within the corresponding coated and pristine silica nanochannels. The pristine silica channels have heights of 3.0, 4.0 and $5.0 \mathrm{~nm}$. The height is defined as the average distance between the inner surfaces of the channel walls. The applied external field corresponds to a value of $9.324 \times 10^{11} \mathrm{~m} / \mathrm{s}^{2}$ which reproduces a pressure equivalent to the higher field applied in the cases with coated channels.

The velocity and density profiles computed for the uncoated channels are presented in Figure 3. The velocity profiles display parabolic shapes with near zero velocity at the solid-liquid interface therefore indicating that the non-slip boundary condition is a good assumption to 
model flow inside the uncoated silica nanochannels. Moreover, the water density profiles display bulk behavior across the entire cross section of the channels, contrasting with the significant water layering observed in the density profile across the corresponding GE coated channels. Using equation 2 , we compute an effective viscosity of $\mu_{e f f}=0.72 \pm 0.02 \mathrm{mPa} \cdot \mathrm{s}$ within the uncoated channels which is in line with values previously reported for $\mathrm{SPC} / \mathrm{E}$ water. ${ }^{38}$ To quantify the flow transport efficiency, we compute the "effective" flow enhancement $\left(\epsilon^{*}\right)$ as the ratio between the flow computed in the coated channels and the flow computed in the bare silica channels. The computed $\epsilon^{*}$ range between 3.2 and 3.7. These values and the corresponding channel heights for each case are listed in Table S4 of the supporting information. From these results, it can be inferred that as the channel height increases, a decrease in $\epsilon^{*}$ is observed, similarly to the observed behavior for $\epsilon$. Nevertheless, values of $\epsilon^{*}$ higher than 3.2 were computed for all the cases indicating that the use of GE monolayers as wall coating in silica nanoslit channels results in a significant decrease of the required energy to transport water through hydrophilic nanoconduits.

The behavior of nanoconfined polar liquids like water depends heavily on the properties of the confining surface, in particular, water molecular orientation and the surface wettability are strongly coupled. ${ }^{36,37}$ To gain further insight into the GE coating effect on the hydrodynamic behavior of the interfacial water within the channel, the average orientation of the water dipole vector is computed. For further details about computing the average water orientation, readers are referred to the supporting information. Figure 4 presents the probability density function (PDF) of the angle between the vector normal to the inner silica surface and the water dipole at different distances from the solid surface. The results presented in Figures $4 \mathrm{~A}$ and $4 \mathrm{~B}$ reveal that there is an interfacial region with oriented water molecules which is thicker for the case without GE coating (pristine silica). Therefore, we find an inverse relation between hydrodynamic slip and the thickness of the interfacial water layer with strongly oriented molecules. Indeed, it implies that the presence of a single GE layer deposited on the inner surface of a silica nanochannel results in significant alteration 
of the strong water molecular ordering typically associated with interfacial water in contact with hydrophilic surfaces. ${ }^{40,41}$

\section{Conclusions}

Atomistic simulations are carried out to evaluate the water flow enhancement induced by a wall coating consisting of monolayer GE in silica nanoslit channels. In these coated channels, a constant slip length of ca. $3.0 \mathrm{~nm}$ is observed. Moreover, flow enhancements ranging from 6.0 to 11.3 are computed by contrasting the flow rates measured from the atomic trajectories against the theoretical flow rates calculated imposing the no-slip boundary condition. Furthermore, when comparing flow rates computed in the coated channels with heights of 4.4, 3.4 and $2.4 \mathrm{~nm}$ against the flow rates computed in the corresponding pristine channels, "effective" flow enhancements ranging from 3.2 to 3.7 are obtained. In order to provide molecular-level insight into the water flow enhancement induced by the GE coating, we compute the average orientation of the water dipole vector at different locations within the solid-liquid interface. The probability density functions indicate that a decrease in the thickness of the interfacial region with structured water is directly related to the flow enhancement measured in the coated channels. Hence, we infer that the presence of the GE sheet increases the fluidity of interfacial water as a result of reduced silica-water binding energy. Overall, our results suggest that the use of GE monolayer as wall coating results in a considerable increase of water flow rates within amorphous silica nanochannels. Hence, deposition of GE layers on inner hydrophilic surfaces in nanoconduits may have significant implications in the development of integrated nanofluidic devices.

\section{Acknowledgements}

E. Wagemann thanks financial support from Centro CRHIAM Conicyt/Fondap Project No 15130015 (Chile). D. Becerra thanks funding from CONICYT through scholarship No 
21181202. H. A. Zambrano acknowledges support from Universidad Tecnica Federico Santa Maria through an DGIIP internal grant. This research was partially funded by CONICYT under FONDECYT project No 11130559. The authors thank computational support from Department of Physics at the Technical University of Denmark and from National Laboratory for High Performance Computing (NLHPC).

\section{Supplementary Material Available}

Molecular Dynamics simulations overview, interaction potentials and models, substrate details, water contact angle measurement, channel filling, temperature profile, binning sampling method, volumetric flow calculation, computed hydrodynamic properties for water confined in coated and uncoated channels, effective enhancement as a function of channel height, orientation function.

\section{References}

(1) Gao, J.; Feng, Y.; Guo, W.; Jiang, L. Nanofluidics in two-dimensional layered materials: inspirations from nature. Chem. Soc. Rev. 2017, 46, 5400-5424.

(2) Goertz, M. P.; Houston, J.; Zhu, X.-Y. Hydrophilicity and the viscosity of interfacial water. Langmuir 2007, 23, 5491-5497.

(3) Ortiz-Young, D.; Chiu, H.-C.; Kim, S.; Voïtchovsky, K.; Riedo, E. The interplay between apparent viscosity and wettability in nanoconfined water. Nature Communications 2013, 4, 1-6.

(4) Kannam, S. K.; Todd, B.; Hansen, J. S.; Daivis, P. J. How fast does water flow in carbon nanotubes? J. Chem. Phys. 2013, 138, 094701. 
(5) Liu, B.; Wu, R.; Baimova, J. A.; Wu, H.; Law, A. W.-K.; Dmitriev, S. V.; Zhou, K. Molecular dynamics study of pressure-driven water transport through graphene bilayers. Phys. Chem. Chem. Phys. 2016, 18, 1886-1896.

(6) Wagemann, E.; Oyarzua, E.; Walther, J. H.; Zambrano, H. A. Slip divergence of water flow in graphene nanochannels: the role of chirality. Phys. Chem. Chem. Phys. 2017, $19,8646-8652$.

(7) Falk, K.; Sedlmeier, F.; Joly, L.; Netz, R. R.; Bocquet, L. Molecular origin of fast water transport in carbon nanotube membranes: superlubricity versus curvature dependent friction. Nano Lett. 2010, 10, 4067-4073.

(8) Tocci, G.; Joly, L.; Michaelides, A. Friction of water on graphene and hexagonal boron nitride from ab initio methods: very different slippage despite very similar interface structures. Nano Lett. 2014, 14, 6872-6877.

(9) Wagemann, E.; Walther, J. H.; Cruz-Chu, E.; Zambrano, H. A. Water Flow in Silica Nanopores Coated by Carbon Nanotubes from a Wetting Translucency Perspective. J. Phys. Chem. C 2019, 123, 25635-25642.

(10) Joseph, S.; Aluru, N. Why are carbon nanotubes fast transporters of water? Nano Lett. 2008, 8, 452-458.

(11) Jung, W.; Kim, J.; Kim, S.; Park, H. G.; Jung, Y.; Han, C.-S. A Novel Fabrication of $3.6 \mathrm{~nm}$ High Graphene Nanochannels for Ultrafast Ion Transport. Advanced Mat. 2008, 29, 1-7.

(12) Akaishi, A.; Yonemaru, T.; Nakamura, J. Formation of Water Layers on Graphene Surfaces. 2017, 2, 2184-2190.

(13) Feng, J.; Guo, Z. Wettability of graphene: from influencing factors and reversible conversions to potential applications. Nanoscale Horiz. 2019, 4, 339-364. 
(14) Chakradhar, A.; Sivapragasam, N.; Nayakasinghe, M. T.; Burghaus, U. Support effects in the adsorption of water on CVD graphene: an ultra-high vacuum adsorption study. Chem. Commun. 2015, 51, 11463-11466.

(15) Shih, C.-J.; Strano, M. S.; Blankschtein, D. Wetting translucency of graphene. Nature Mat. 2013, 12, 866-869.

(16) Hong, G.; Han, Y.; Schutzius, T. M.; Wang, Y.; Pan, Y.; Hu, M.; Jie, J.; Sharma, C. S.; Muller, U.; Poulikakos, D. On the mechanism of hydrophilicity of graphene. Nano Lett. 2016, 16, 4447-4453.

(17) Ashraf, A.; Wu, Y.; Wang, M. C.; Yong, K.; Sun, T.; Jing, Y.; Haasch, R. T.; Aluru, N. R.; Nam, S. Doping-Induced Tunable Wettability and Adhesion of Graphene. Nano Lett. 2016, 16, 4708-4712.

(18) Rafiee, J.; Mi, X.; Gullapalli, H.; Thomas, A. V.; Yavari, F.; Shi, Y.; Ajayan, P. M.; Koratkar, N. A. Wetting transparency of graphene. Nature Mat. 2012, 11, 217-222.

(19) Shih, C.-J.; Wang, Q. H.; Lin, S.; Park, K.-C.; Jin, Z.; Strano, M. S.; Blankschtein, D. Breakdown in the wetting transparency of graphene. Phys. Rev. Lett. 2012, 109, 176101.

(20) Lu, J.-Y.; Olukan, T.; Tamalampudi, S. R.; Al-Hagri, A.; Lai, C.-Y.; Al Mahri, M. A.; Apostoleris, H.; Almansouri, I.; Chiesa, M. Insights into graphene wettability transparency by locally probing its surface free energy. Nanoscale 2019, 11, 7944-7951.

(21) Ramos-Alvarado, B.; Kumar, S.; Peterson, G. On the wettability transparency of graphene-coated silicon surfaces. J. Chem. Phys. 2016, 144, 014701.

(22) Raj, R.; Maroo, S. C.; Wang, E. N. Wettability of Graphene. Nano Lett. 2013, 13, $1509-1515$. 
(23) Hung, S.-W.; Hsiao, P.-Y.; Chen, C.-P.; Chieng, C.-C. Wettability of Graphene-Coated Surface: Free Energy Investigations Using Molecular Dynamics Simulation. J. Phys. Chem. C 2015, 119, 8103-8111.

(24) Ramos-Alvarado, B.; Kumar, S.; Peterson, G. Wettability transparency and the quasiuniversal relationship between hydrodynamic slip and contact angle. Appl. Phys. Lett. 2016, 108, 074105 .

(25) Xie, Q.; Alibakhshi, M. A.; Jiao, S.; Xu, Z.; Hempel, M.; Kong, J.; Park, H. G.; Duan, C. Fast water transport in graphene nanofluidic channels. Nature Nanotechnol. 2018, 1 .

(26) Jin, Y.; Tao, R.; Li, Z. Understanding flow enhancement in graphene-coated nanochannels. Electrophoresis 2019, 40, 859-864.

(27) Sam, A.; Kannam, S. K.; Hartkamp, R.; Sathian, S. P. Water flow in carbon nanotubes: The effect of tube flexibility and thermostat. J. Chem. Phys. 2017, 146, 234701.

(28) Lee, T.; Charrault, E.; Neto, C. Interfacial slip on rough, patterned and soft surfaces: A review of experiments and simulations. Adv. Coll. Interf. Sci. 2014, 210, 21-38.

(29) Walther, J. H.; Jaffe, R.; Halicioglu, T.; Koumoutsakos, P. Carbon nanotubes in water: Structural characteristics and energetics. J. Phys. Chem. B 2001, 105, 9980-9987.

(30) Berendsen, H. J. C.; Grigera, J. R.; Straatsma, T. P. The missing term in effective pair potentials. J. Phys. Chem. 1987, 91, 6269-6271.

(31) Guissani, Y.; Guillot, B. A numerical investigation of the liquid-vapor coexistence Curve of Silica. J. Chem. Phys. 1996, 104, 7633-7644.

(32) Tsuneyuki, S.; Tsukada, M.; Aoki, H.; Matsui, Y. First-principles interatomic potential of silica applied to molecular dynamics. Phys. Rev. Lett. 1988, 61, 869-874. 
(33) Zambrano, H.; Walther, J. H.; Jaffe, R. Molecular dynamics simulations of water on a hydrophilic silica surface at high air pressures. J. Mol. Liq. 2014, 198, 107-113.

(34) Werder, T.; Walther, J. H.; Jaffe, R. L.; Halicioglu, T.; Koumoutsakos, P. On the water-graphite interaction for Use in MD simulations of graphite and carbon nanotubes. J. Phys. Chem. B 2003, 10\%, 1345-1352.

(35) Zhang, Z.; Li, T. A molecular mechanics study of morphologic interaction between graphene and $\mathrm{Si}$ nanowires on a $\mathrm{SiO} 2$ substrate. Journal of Nanomat. 2011, 2011, 1-7.

(36) Thomas, J. A.; McGaughey, A. J. Reassessing fast water transport through carbon nanotubes. Nano Lett. 2008, 8, 2788-2793.

(37) Wu, K.; Chen, Z.; Li, J.; Xu, J.; Dong, X. Wettability effect on nanoconfined water flow. Proc. Natl. Acad. Sci. USA 2017, 114, 3358-3363.

(38) González, M. A.; Abascal, J. L. The shear viscosity of rigid water models. J. Chem. Phys. 2010, 132, 096101.

(39) Choi, C.-H.; Westin, K. J. A.; Breuer, K. S. To slip or not to slip: Water flows in hydrophilic and hydrophobic microchannels. ASME 2002 International Mechanical Engineering Congress and Exposition. 2002; pp 557-564.

(40) Giovambattista, N.; Debenedetti, P. G.; Rossky, P. J. Effect of Surface Polarity on Water Contact Angle and Interfacial Hydration Structure. J. Phys. Chem. B 2007, $111,9581-9587$.

(41) Karna, N. K.; Crisson, A. R.; Wagemann, E.; Walther, J. H.; Zambrano, H. A. Effect of an external electric field on capillary filling of water in hydrophilic silica nanochannels. Phys. Chem. Chem. Phys. 2018, 20, 18262-18270. 

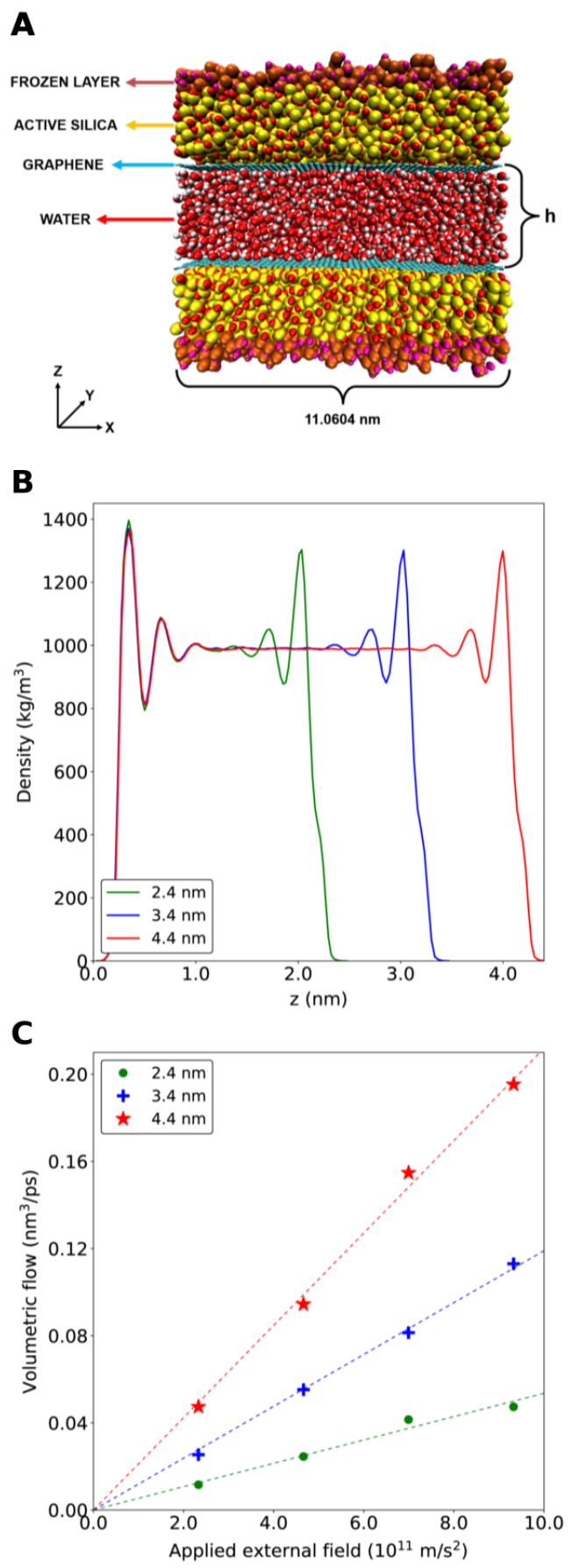

Figure 1: A) Snapshot of the GE coated silica nanoslit channel. Water is confined between two parallel amorphous silica slabs. In each slab the inner surface is coated with a graphene sheet. $h$ corresponds to the channel height and is defined as the average distance along the $z$ direction between the two GE sheets. B) Density profiles for different channel heights and C) volumetric flow rates measured for different channel heights. 

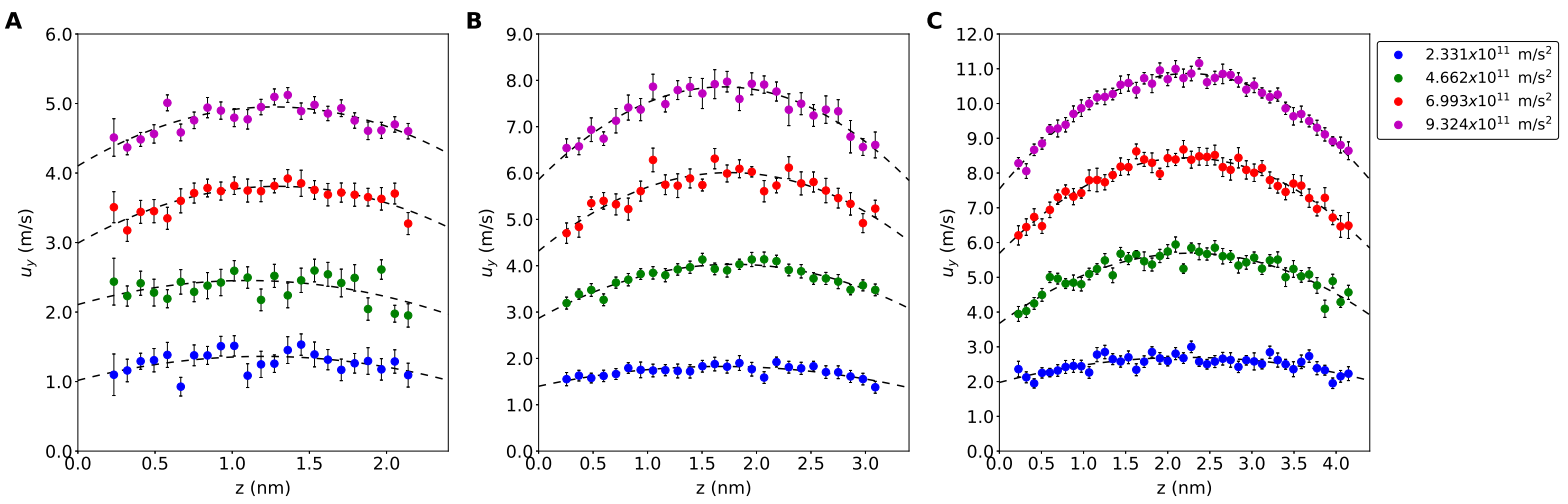

Figure 2: Water velocity profiles for different applied external fields within the graphene coated silica channels with heights of A) $2.2 \mathrm{~nm}$, B) $3.3 \mathrm{~nm}$ and C) $4.4 \mathrm{~nm}$. The black dashed line depicts a parabolic fit to the measured velocity distribution across the channel.
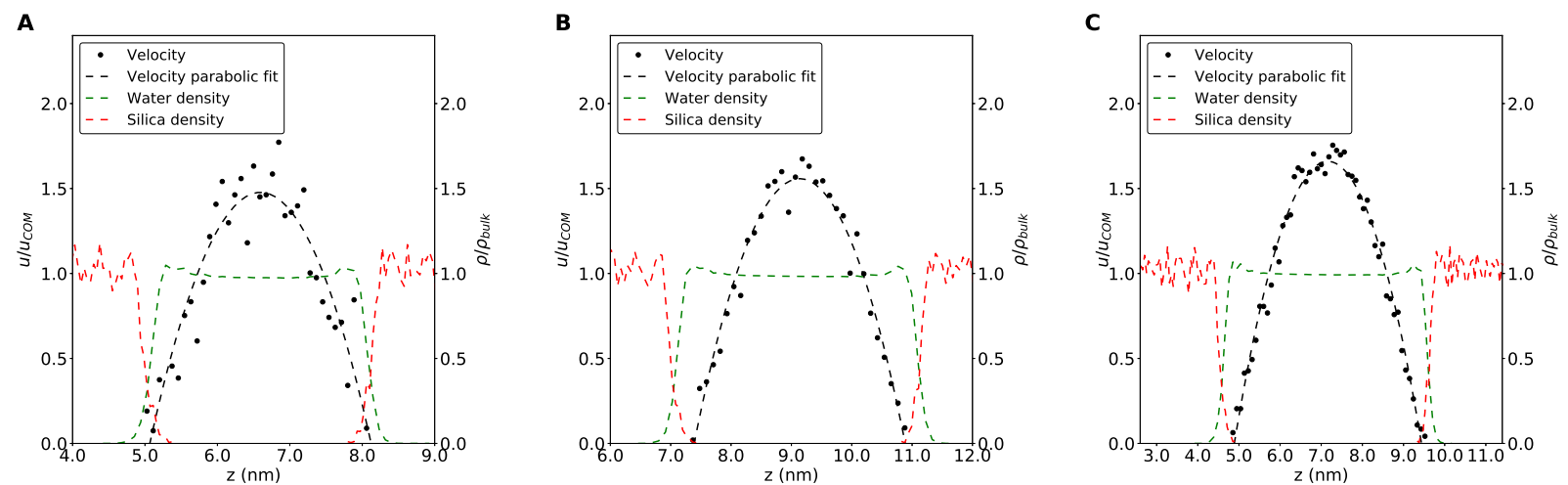

Figure 3: Normalized velocity and density profiles for water flow inside an uncoated silica channel with heights of A) $3.0 \mathrm{~nm}$, B) $4.0 \mathrm{~nm}$ and C) $5.0 \mathrm{~nm}$. 
A

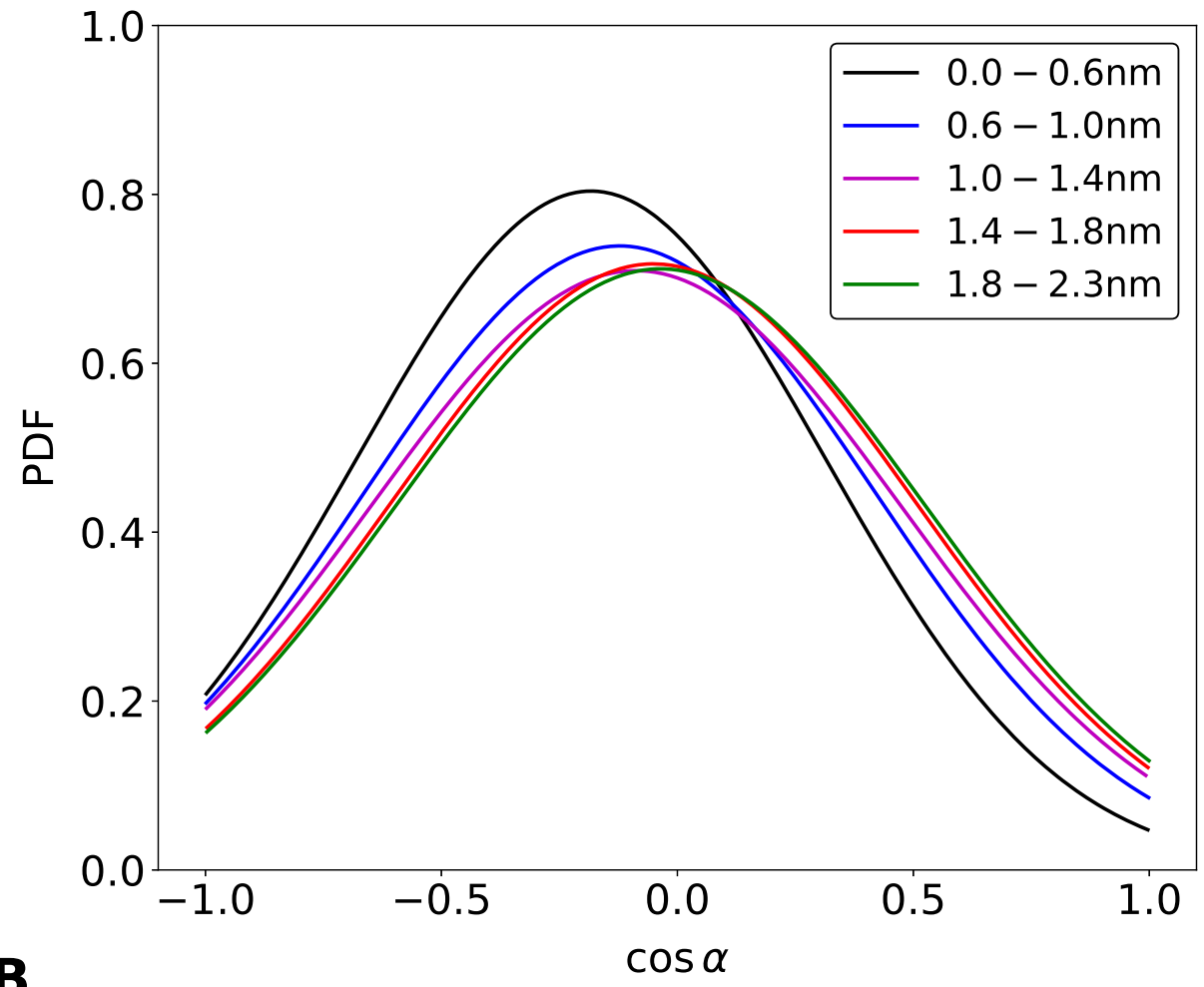

B

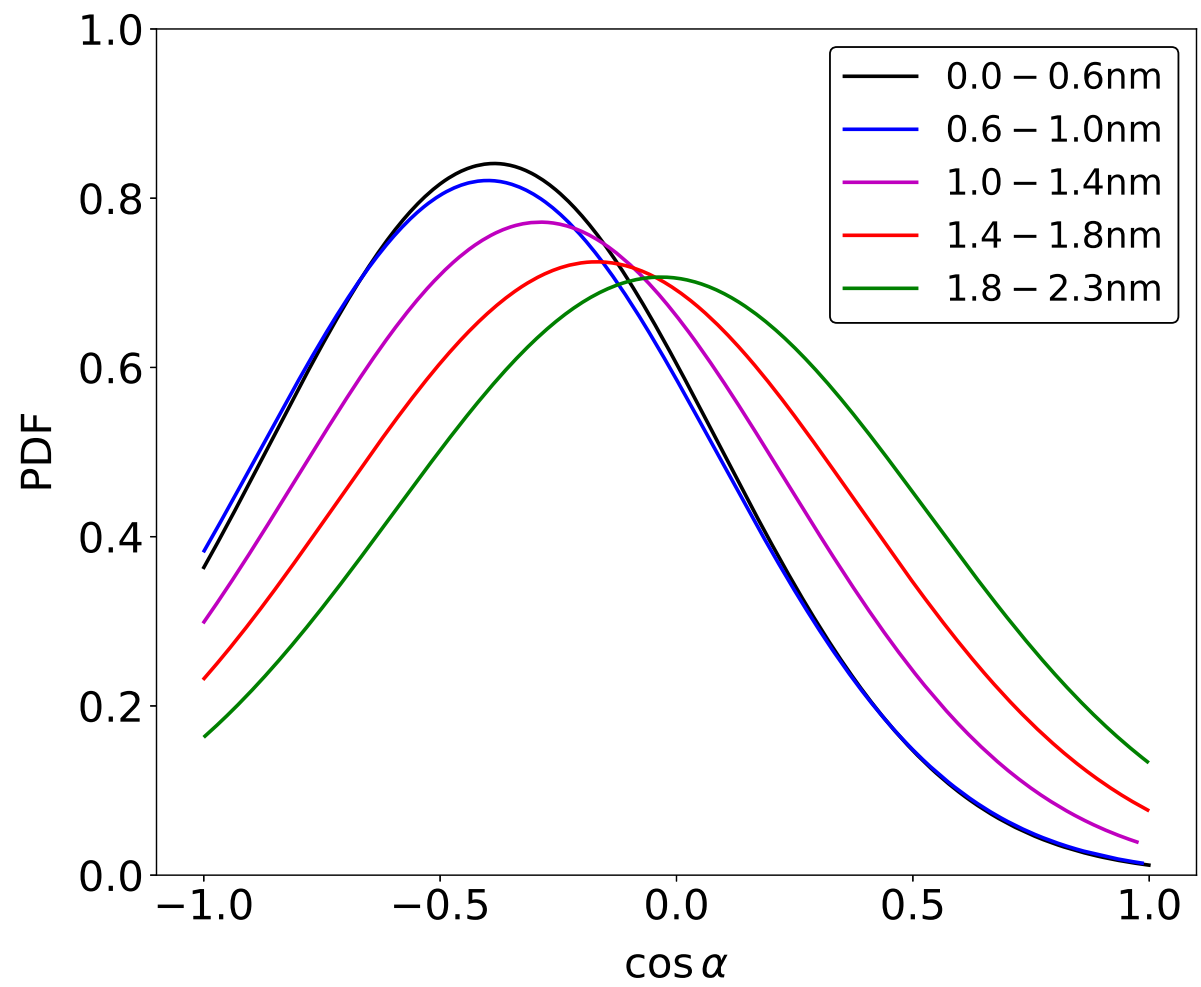

Figure 4: Probability distribution function of water dipole moment at different distances from the wall surface. a) Graphene coated channel. b) Channel without wall coating. 